\title{
The Argument from Pain: A new Argument for Indirect Realism
}

(Penultimate version; published in: Grazer Philosophische Studien 93 (2016), 106-129)

Abstract: I will put forward and defend a new argument for indirect realism which I call the argument from pain. The argument is akin to a well-known traditional argument to the same end, the argument from hallucination. As the latter, it contains one premise stating an analogy between veridical perceptions and certain other states and one premise stating that those states are states of acquaintance with sense-data. The crucial difference is that the states that are said to be analogous to veridical perceptions are pain-states instead of hallucinations. This difference makes the argument from pain immune to the standard objections against the argument from hallucination.

\section{The Argument from Pain}

According to indirect realism concerning visual experiences, the subject of every visual experience is immediately acquainted with nothing but one or more subjective, mental particulars, so-called sense-data, which represent (in almost all cases) the objective, physical objects the experience is about. Today, this theory is hardly taken seriously any more. To my mind, this dismissive attitude is unwarranted. In particular, it seems to me that most current authors underestimate the force of the arguments on behalf of indirect realism. In this paper I will put forward and defend one such argument. I call it the argument from pain. It runs as follows:

(P1) Visual experiences are analogous to pain-states in all critical respects.

(P2) Being in a pain-state is to be acquainted with nothing but a sense-datum, the pain.

(C) Being in a visual experience is to be acquainted with a sense-datum as well. 
Henceforth I call (P1) the analogy-thesis and (P2) the pain-thesis. Two terms may ask for explanation. The critical respects referred to in (P1) are those respects that are critical for the truth value of the conclusion of the argument. And pain-states are the mental states subjects are in when they are in pain. (This stipulation prevents us from preempting the non-trivial question of whether what we call pains in everyday discourse are the mental states we are in when we are in pain or rather the objects of these states. Its utility will become apparent soon.)

As everyone at home in the philosophy of perception will realize, the general structure of the argument from pain is taken over from another traditional argument to the same end, the so called argument from hallucination. Here is an (incomplete) version of this argument highlighting this structural parallelism (for a recent elaborated version see Thompson 2008):

(P1) Hallucinations and veridical perceptions are states of the same fundamental kind.

(P2) Being the subject of a hallucination means to be acquainted with nothing but sensedata.

(C) Having a veridical perception is to be acquainted with nothing but sense-data as well.

Both arguments are composed of one premise stating a relevant kinship between veridical perceptions and certain other states and one premise stating that those states consist of (nothing but) the acquaintance with sense-data. But while in the argument from hallucination the states that are said to be analogous to veridical perceptions are visual states as well, namely hallucinations, in the argument from pain they are pain-states. This difference carries a significant advantage for the argument from pain over the argument from hallucination: While the analogy-thesis is, at worst, slightly more difficult to defend than (P1) of the argument from hallucination, the pain-thesis is considerably easier to defend than (P2) of the argument from hallucination. 
The structure of the paper corresponds to a great extend to the structure of the argument from pain. In section 2 I defend the analogy-thesis. In section 3 I first defend the pain-thesis and then try to bring out the real force of the argument somewhat more perspicuously. I end with a brief conclusion.

\section{The Analogy-Thesis}

Visual experiences are analogous to pain-states in all critical respects.

In general it is useful to distinguish between two kinds of considerations about mental states: considerations from the first- and from the third-person-perspective. ${ }^{1}$ Corresponding to this distinction, there is a distinction between two parts of the analogy-thesis:

First-person analogy-thesis: Considered from the first-person-perspective, visual experiences and pain-states are analogous in all critical respects.

Third-person analogy-thesis: Considered from the third-person-perspective, visual experiences and pain-states are analogous in all critical respects.

I consider both theses in turn, beginning with the first-person analogy-thesis. Each time I proceed as follows: Firstly, I set forth the relevant features of visual experiences. Then, I consider whether they are analogous to the relevant features of pain-states. Since space does not allow for defending every single claim pertinent to this reasoning, I offer such a defense only for those claims which I take to be most controversial.

\footnotetext{
${ }^{1}$ I take this distinction to be sufficiently well understood to make use of it throughout the following discussion.
} 


\section{The first-person analogy-thesis}

For convenience, I will talk of considerations from the first-person-perspective as phenomenological considerations, of the features 'accessible' from the first-personperspective alone as phenomenological features and of the sum of these features as the phenomenology of the relevant mental state. To my mind, the phenomenology of visual experiences is mainly determined by two closely related features, which I shall call relationality and transparency. Here is a compact characterization of both as exemplified in visual experiences:

Relationality: In any visual experience the subject of the experience is immediately acquainted with one or more instances of sensible properties.

Transparency: The instances of sensible properties the subject of an experience is immediately acquainted with fully exhaust the way it is like for the subject to be in this visual experience.

I assume without further argument that visual experiences do indeed have these features ${ }^{2}$ and confine myself to some explanatory remarks. Firstly, since sensible properties are properties of the objects of visual experiences and not of the experiences themselves, the transparency of visual experiences implies that the way it is like for a subject to be in a visual experience is determined by properties of the objects of the experience, and not by properties of the experience itself. Secondly, both features under consideration are phenomenological features. That is, the claim that visual experiences have these features bears no ontological implications beyond the fact that visual experiences have the phenomenology they have. This carries a

\footnotetext{
${ }^{2}$ I defend this assumption elsewhere (see [author's name] (forthcoming)).
} 
consequence which is of some importance for my investigation: Since the phenomenology of visual experiences is neutral regarding the question of whether the sensible properties subjects of visual experiences are acquainted with are properties of physical objects or properties of sense-data, the fact that visual experiences are relational and transparent is neutral in this respect too. ${ }^{3}$

What about pain-states? Do they have phenomenological features analogous to those of visual experiences? In this case something along the following lines would be true:

Relationality: In any pain-state the subject of the state is immediately acquainted with a specific kind of uncomfortable quality. ${ }^{4}$

Transparency: The instance of the uncomfortable quality the subject of a pain-state is immediately acquainted with fully exhausts the way it is like for the subject to be in this pain-state.

Initially, there is strong phenomenological evidence for both claims. Take any pain-state you are in when you have a pain in your ankle. To be in this state means for you to be immediately acquainted with an uncomfortable quality that is instantiated in what you take to be your ankle. And whatever it is that you take to be your ankle - whether it is indeed your ankle or whether it is a sense-datum representing your ankle - it is an object of your pain-state. It is not the state itself. Hence, the uncomfortable quality you are acquainted with is not a property of the state you are in. It is a property of the object of this state. Moreover, the relevant

\footnotetext{
${ }^{3}$ This is not in line with the standard way to understand these features. In particular, transparency is commonly characterized in a way, according to which it is incompatible with Indirect Realism (see e.g. Harman 1990, Martin 2002 or Rowlands 2001). To my mind, these characterizations are inadequate insofar as they exceed the limits of phenomenology (see also [author's name] (forthcoming)).

${ }^{4}$ Note that this is not meant to imply that the relevant quality is intrinsically or essentially uncomfortable.
} 
uncomfortable quality you are acquainted with fully exhausts the phenomenal quality of your pain-state. No other property, especially no property of this state itself, contributes to its phenomenal quality. So it seems that pain-states are indeed relational and transparent in the sense specified above.

This conclusion, however, flies in the face of a view that was orthodox for a long time and is still widespread. I shall call it the orthodox view - even though it may have lost the status of orthodoxy since. One way to characterize this view is this:

(1) No pain-state is directed towards any object different from itself. ${ }^{5}$ (Note: Here, difference does not imply independence.)

(2) The uncomfortable quality a subject of a pain-state is acquainted with is a property of this state itself.

(3) The phenomenal consciousness of a pain-state consists in the subject's acquaintance with this property. ${ }^{6}$

\footnotetext{
${ }^{5}$ Here, difference does not imply independence. Hence, even sense-data would be classified as objects different from pain-states.

${ }^{6}$ Some opponents of the claim that pain-states are relational and transparent may find assumptions (2) and (3) not mandatory. Why not just deny, they may ask, that the subject of a pain-state is, in virtue of her being in this state, acquainted with anything at all, be it an object different from the state or the state itself? I take it that a view along these lines is unable in principle to account for the phenomenal consciousness of pain-states. That a state is phenomenally conscious means, according to the common nagelian way of speaking, that it is somehow for a subject to be in this state. But, it is hard to understand how it could be somehow for the subject of a mental state to be in this state if it were not acquainted with anything at all. Phenomenal consciousness seems to me just inconceivable without some kind of acquaintance-like structure. The relevant acquaintance may be outwardly or inwardly directed, but it has to be there. If this is right, every opponent of the claim of the transparency of painstates willing to do justice to the phenomenal consciousness of pain-states must also endorse the second and third assumptions of the orthodox view.
} 
We just saw that there is strong phenomenological evidence speaking against it. But in the light of its lasting popularity, one is well advised to back this evidence through further arguments. Here are three arguments to this end.

(1) Apparently, pain-states have a spatial structure. The uncomfortable quality one is acquainted with when having a pain is, in some sense, located in one's body. If one has a pain in one's ankle (tooth...), it is, in some sense, located in one's ankle (tooth...). And, what is more, it has this location essentially. It is not just projected onto the relevant location. It is literally there. This is confirmed by one's inability to imagine it as not being located at a specific position in one’s body. As Brewer has felicitously put it: "Spatiality cannot be stripped away from bodily sensation without significant loss.“ (Brewer 1995, p. 304) To be sure, the exact nature of this spatial location is not obvious from the outset (that is the reason for the qualification 'in some sense' above). The most natural assumption is that the space the uncomfortable quality is located in is just the physical space with the physical body in it. But some authors feel compelled to introduce a sui generis phenomenal space in which minddependent entities like sense-data are located (see e.g. Castañeda 1977 or Casullo 1986). In this case the relevant uncomfortable quality would be located in this sui generis phenomenal space. But no matter which proposal one prefers, both contradict the assumption, entailed by the orthodox view, that a subject's acquaintance with a relevant uncomfortable quality is an introspective acquaintance with its own pain-state.

(2) When one is in a pain-state one can (under fairly normal conditions) draw one's attention to the uncomfortable quality one is acquainted with in being in this state. On the orthodox view this uncomfortable quality is a property of the pain-state itself. Therefore, it is a condition of the truth of the orthodox view that the subject of a pain-state can draw its attention to its own pain-state. This condition, however, is incompatible with an initially plausible principle, the principle that one can draw one's attention only to objects (or 
properties of objects) of one's mental states, but not to those mental states themselves. Hence, the proponent of the orthodox view is committed to rejecting this principle, which is, given its initial plausibility, an uncomfortable position. But even if one is prepared to accept that there are cases that does not conform to the principle, the question remains whether cases of painstates are among these cases. Our intuitions about concrete cases of pain-states strongly suggest otherwise. Apparently, the attention one draws to the uncomfortable quality one is acquainted with in having, say, a pain in ones ankle is an outward-directed one - an attention directed on a property of ones ankle and not on one's mind (for a similar argument see Hill 2006, p. 89). ${ }^{7}$

(3) The third argument is along similar lines. The following two claims seem to be true: (i) The subject of a pain-state can (under fairly normal conditions) make a demonstrative reference to the uncomfortable quality it is acquainted with in being in this state. (ii) A demonstrative reference can be made only to objects or properties of objects of mental states, never to the mental states themselves. The conjunction of both claims, however, is incompatible with the orthodox view. It implies that the uncomfortable quality the subject of a pain-state is acquainted with is a property of an object of this state, not of the state itself, as the orthodox view says. So, the proponent of the orthodox view is again in an uncomfortable position. She has to reject one of the two seemingly true claims.

It is not obvious, however, that there are no good reasons to do so. Note that while it is beyond dispute that one can, on occasion, make true assertions in uttering sentences like "This pain is annoying" or "That pain is radiating into my shoulder" it is not beyond dispute that this fact supports the claims under consideration. For one thing, the proponent of the orthodox

\footnotetext{
${ }^{7}$ To some it may seem odd to read these sentences in a paper that is announced as a defense of Indirect Realism. Are sense-data not part of the mind? Well, true, in a sense they are. They are mind-dependent objects. But this does not support the assumption that attending to them is a kind of introspection. This would be the case only if they were properties of mental states, which they are not.
} 
view might try to show that, contrary to first appearance, such assertions do not contain genuine demonstrative references. The most promising way to do this is to claim that phrases like 'this pain' or 'that pain' in the relevant assertions are roughly synonymous with 'my pain' or 'the pain I actually have', which are not demonstrative expressions. ${ }^{8}$ However, to see that this cannot be right just consider a sentence like “This pain is stronger than that pain”. According to the proposed interpretation, it is to be paraphrased as "My pain is stronger than my pain”. But this is inadequate for obvious reasons. For another thing, the proponent of the orthodox view might contest the second claim that a demonstrative reference can be made only to objects (or properties of objects) of mental states, never to the mental states themselves. She might point out that if one has e.g. a visual experience of something red, one can say things like: "This experience is (as) of something red” which seem to show that one is very well able to refer to once experiences with demonstrative expressions. This, however, is of no help to the proponent of the orthodox view. For one thing, we have already learned that the bare fact that one utters such sentences fails to establish that one is thereby making genuine demonstrative references to one's experiences. And, more importantly, even if we accepted the latter assumption, this would not undermine the argument against the orthodox view. Think about occasions at which we are able to make assertions like "This experience is of something red”. Apparently, among the conditions that enable us to make these allegedly demonstrative references to our experiences are just those conditions that enable us to make demonstrative references to the objects of these experiences. To be more precise, if we can demonstratively refer to our experiences at all, we can do so only in virtue of our being

\footnotetext{
${ }^{8}$ Another, more radical, possibility is to insist that uttering the sentences in question is nothing but a kind of expressive behavior, roughly on a par with groaning or screaming. This view is well known as Expressivism. If it were correct, the utterances in question would not express any kind of thought apt for being true at all. However, Expressivism is not only highly implausible, at least as a general view, it also harmonizes very badly with the spirit of the orthodox view.
} 
acquainted with the objects of these experiences. And the same holds for the allegedly demonstrative references to our pain-states. They rest on our acquaintance with the objects of these states. And since the obtaining of such an acquaintance is just what is denied by the orthodox view, the objection against the second claim fails to support this view anyway.

I concede that none of these arguments against the orthodox view is entirely conclusive. But taken together and before the background of the phenomenological evidence put forward above they give us very good reason to abandon this view and to accept the first-person analogy-thesis - or so I assume.

This result puts me in the position to make a crucial terminological stipulation: The uncomfortable qualities subjects are acquainted with when they are in pain-states be pains. Note that this stipulation is neutral regarding the precise ontological status of pains. Moreover, I leave it open, at least for the moment, whether or not the relevant qualities would be pains even if no subject were acquainted with them. The reasons for this will become apparent soon.

\section{The third-person analogy-thesis}

I assume that the most striking third-personal feature of visual experiences is the distinction between veridical and non-veridical experiences. The standard definition of these notions is something like this: A visual experience is veridical if and only if (i) there are objects for which it is true that they appear in the experience (i.e. for which it is true that the experience is about them) and (ii) these objects appear in it as they really are. If either the second or both conditions are not met, the experience is non-veridical. In the former case it is an illusion, in the latter a hallucination. ${ }^{9}$ This definition, however, puts us in a somewhat delicate situation.

\footnotetext{
${ }^{9}$ Note that this is not meant to rule out that there is a sense in which it is true to say that something appears (as something) in a hallucination. Condition (i) is to be understood as quantifying over concrete, existing objects.
} 
On the one hand, it will not be of much help so long as we do not have at least a rough idea of what conditions (i) and (ii) amount to. On the other hand, there is disagreement between proponents of different theories of visual experiences precisely on this matter and, on pain of weakening our argument, we should try to remain neutral regarding different theories of visual experiences at this point. Fortunately, there is a way out of this predicament. For our purposes it is sufficient to depict those features of visual experiences which are relevant for the question of the analogy or disanalogy with pain-states. And these are precisely not the features which are the subjects of controversy among the proponents of different theories of visual experiences. More specifically, the following approximate explication of the core notion of an object's appearing as it really is should be acceptable for all parties and serves our purposes perfectly well:

An object appears in a particular experience as it really is, only if its sensible properties stand in a relation to this experience which meets the following conditions:

(a) It is one of the sensible properties' of the object bringing about the relevant experience in a specific way.

(b) It obtains if the conditions relevant for visual experiences are fairly normal.

(c) It enables the relevant subject to act properly with regard to the relevant objects.

On this basis we can explicate the other relevant notions as well: If condition (a), but neither (b) nor (c) is met, the object appears as different from how it really is. And if none of the conditions (a) - (c) is met there is no object appearing at all.

And I concede that talk of appearing does not in itself entail a commitment to concrete, existing objects for which it is true that they appear to someone. 
Before I proceed, let me emphasize a point that is of some relevance for the purposes of our investigation: That the distinction between veridical and non-veridical experiences is a distinction from the third-person-perspective means that it does not affect the phenomenology of the relevant experiences. That is, non-veridical experiences have the very same phenomenological features as veridical experiences. They are relational and transparent, just as the latter. This fact bears two important consequences. For one thing, all ontological implications eventually resulting from an experience's having these features hold for cases of non-veridical experiences as well. ${ }^{10}$ For another thing, the fulfillment of conditions (i) and (ii) of the initial definition is not among these implications. In order for an experience to be relational and transparent, neither condition has to be met.

With this in mind let us come to pain-states. Is there a distinction between different painstates analogous to the distinction between veridical and non-veridical experiences? The answer to this is again affirmative.

For ordinary cases of pain-states it is true that there is a relation between damage to the body of the subject of the relevant state and the state itself such that

(a) it (the relation) is one of the damage's to the body somehow bringing about the pain-state in a certain way,

(b) it obtains if the conditions relevant for pain-states are fairly normal and

(c) it enables the subject to act properly with regard to the relevant body-part and its damage.

\footnotetext{
${ }^{10}$ Note, however, that this fact alone does not imply that all three kinds of visual experiences are of the same metaphysical kind. Whether the latter is the case, depends on the question whether phenomenological kinship is compatible with a difference in metaphysical kind - a question hotly disputed among proponents of different theories of visual experiences.
} 
Furthermore, there are both cases of pain-states in which condition (a) but neither (b) nor (c) is met, and cases of pain-states in which none of the conditions (a) - (c) is met. Cases of the former kind are typically case in which the subject has the relevant body-part, but it is not damaged in the relevant way. And cases of the latter kind are typically cases in which the subject does not even have the relevant body-part (so called phantom-limb pains). All this strongly suggests that there are indeed pain-states which are analogous in all critical respects to veridical, illusory and hallucinatory visual experiences. ${ }^{11}$

To be sure, just as in the case of visual experience, there will be different opinions about the exact nature of the relation under consideration. But, as indicated above, there is no reason to assume that the nature of this relation is fundamentally different in cases of visual experiences and in cases of pain-states. As a consequence, the fact that both kinds of states meet our preliminary characterization of the relevant distinction is reason enough to accept the thirdperson analogy-thesis.

Hence, since the first-person analogy-thesis turned out to be true as well, we are entitled to take the analogy-thesis, the first premise of the argument from pain, to be confirmed.

\section{The Pain-Thesis}

Being in a pain-state is to be acquainted with a sense-datum, the pain.

In this section I defend the second premise of the argument from pain, the Pain-thesis. I begin by outlining the accounts of pain-states compatible with the results reached in the previous section. Then I establish an important principle about pains and pain-states and show that in

\footnotetext{
${ }^{11}$ One might object that we never speak of pain-states as illusory or hallucinatory or of something appearing as something in pain-states. This is true, but does not contradict our result. For, what we would say in speaking this way may be true nonetheless.
} 
order to do justice to this principle we have no choice, but to accept the account of pain-states that entails the pain-thesis, namely indirect (pain-)realism (see below).

The previous section was dedicated to the defense of the analogy-thesis. Hence, it is no surprise that the accounts of pain-states compatible with the results of this section are analogous to some plain-old accounts of visual experiences. Alluding to these analogies, I shall call them naïve pain-realism, pain-intentionalism and indirect pain-realism. The crucial difference between these accounts concerns the ontological status of pains.

According to naïve pain-realism, pains are

(i) physical, public properties (presumably damage to body-parts) that are

(ii) constituents of the respective pain-states themselves (that is, a pain-state cannot take place in the absence of a pain).

According to pain-intentionalism, pains are

(i) physical, public properties (presumably damage to body-parts) that are

(ii) represented by pain-states (that is, a pain-state can take place in the absence of a pain).

According to indirect pain-realism, pains are

(i) properties of subjective mental particulars and, in addition to that,

(ii) representations of physical, public properties (presumably damage to body-parts). ${ }^{12}$

\footnotetext{
${ }^{12}$ This characterization departs slightly from the usual understanding of this position, according to which pains are just sense-data instead of properties of sense-data. However, due to the ontological 'thinness' of sense-data, nothing substantial hinges on this difference and my characterization makes it easier to highlight the analogy with visual sensible properties like (sensible) redness (see below).
} 
As is highlighted in nearly all works on pains and pain-states (see, for a representative example, Hill 2006), our everyday talk about pain-states is (inter alia) guided by a principle that has no obvious analog in our everyday talk about visual experiences. I shall call it the principle of the mind-dependence of pain (henceforth: PMDP).

The principle of the mind-dependence of pain: For any subject it is impossible that (i) it is for it as if it has a pain while, in fact, it does not have a pain, and that (ii) it does not have a pain while it is for it as if it has a pain.

Correctly understood, this principle will provide the criterion we need for choosing the right account of pain-states. But how is it understood correctly? The answer to this question is by no means obvious. It has been pointed out by different authors that our everyday use of the term 'pain' tends to be ambiguous between referring to pains and to pain-states (see e.g. Harman 1990 or Tye 2006a). And, corresponding to this alleged ambiguity, the principle allows for two significantly different readings.

The first reading complies with our own terminology. That is, "pain” stands for pain. I shall call this the literal reading. In the second reading, "pain” stands for pain-state. In this reading, which I shall call the non-literal reading, the principle amounts to this:

For any subject it is impossible that (i) it is for it as if it has a pain while, in fact, it is not in a pain-state and that (ii) it is not in a pain-state while it is for it as if it has a pain.

Before we address the question of how the principle is to be read, let us have a closer look at the main implications of both readings. In the non-literal reading the principle comes close to a triviality, for, as nearly everyone will acknowledge, that it is for a subject as if it has a pain 
is a necessary as well as a sufficient condition for that subject's being in a pain-state. Consequently, in its non-literal reading the principle does not bear any problematic or surprising implications, which is, no doubt, one reason for the tendency among philosophers to endorse this reading of the principle.

Things are quite different, however, with respect to the literal reading of the principle: If the principle in this reading were true, pains would have a very remarkable feature. They would exist - whatever their existence may consist in - if and only if the states which are about them, namely pain-states, exist. As an illustration, take the following pair of situations:

$\mathrm{SP}_{1}$ : James has a phantom-limb pain in his right knee. That is, it is for him as if he has a pain in his right knee, even though he does not have a right knee any more (his right leg has been amputated, say).

$\mathrm{SP}_{2}$ : James' right knee is injured in a way such that if the conditions were normal, it would be for him as if he had a pain in his right knee. But since the conditions are not normal, it is not this way for James.

Given the truth of the PMDP in its literal reading, the following is true: $\mathrm{In}_{\mathrm{SP}_{1}}$ there is a pain that James feels since it is for James as if there is one. In $\mathrm{SP}_{2}$ there is no pain James feels since it is not for James as if there is one. This would mark a fundamental disanalogy between pains and visible properties like ordinary, physical redness. Consider, for comparison, the following pair of situations:

$\mathrm{SV}_{1}$ : James undergoes a hallucination as of a red wall. That is, it is for him as if there is a red wall before him while, in fact, there is no wall before him. 
$\mathrm{SV}_{2}$ : There is a red wall such that, if the conditions were normal, it would be for James as if there were a red wall before him. But since the conditions are not normal, it is not this way for James.

In $\mathrm{SV}_{1}$ there is no physical redness before James even though it is for him as if there is physical redness before him. In $\mathrm{SV}_{2}$ there is physical redness before James even though it is not for him as if there is physical redness before him. The importance of this disanalogy is evident. It is an implication of the analogy-thesis that any pain has to have an analog in every situation of a visual experience. And the obvious candidates to play this role are visible properties like ordinary, physical redness. But if the disanalogy just mentioned obtained, these properties could not play this role.

This said, we have a fairly solid understanding of both readings of the PMDP. But in which of these readings is the principle true? It certainly is in the non-literal reading, but is it also true in the literal reading? It will come as no surprise that my answer to this question is in the affirmative. To my mind, when we use the term 'pain' in our everyday discourse, we express, often or even always, a concept that is determined by the PMDP in its literal reading.

However, giving arguments for this answer that go beyond repeating the principle with emphasis is not an easy matter. Here is my best try: The evidence for the principle is provided by a combination of intuitions about our everyday concept of pain and phenomenological observations of pains and pain-states. First to the conceptual intuitions: What is the basis on which we settle questions regarding the occurrence of pains in given situations? Apparently, our answers to such questions rest exclusively on our information about how it is for the relevant subjects to be in the states they are in in the situations under consideration. If it is for a subject as if it has a pain, as it is with James in $\mathrm{SP}_{1}$, there is a pain and if it is not this way for it, as it is with James in $\mathrm{SP}_{2}$, there is no pain. Given this much is conceded, the crucial question is: Is the fact that it is for a subject as if it has a pain explainable without assuming 
the existence of a pain? If this were true, there would be no reason to believe in PMDP in the literal reading. And this is exactly the answer commonly given. To my mind, however, this answer is fallacious. Here the aforementioned phenomenological observation comes in. Ask yourself: What does it mean that it is for a subject as if it has a pain? A common answer, incorporated in the orthodox view, is this: It just means that the subject is in a pain-state with the phenomenal quality specific for pain-states, whereas this, in turn, means that the subject is somehow acquainted with its own pain-state. This answer avoids the assumption of the existence of pains. But it is to be rejected. For, as we have revealed in the previous section, the second part of the answer is incompatible with the transparency of pain-states, that is, with the fact that the phenomenal quality of pain-states is exhausted by the uncomfortable property the subject is acquainted with. So, what, then, is the correct answer? It is entailed by the very same fact. Given the transparency of pain-states, as just stated, it cannot be somehow for a subject to be in a pain-state without there being an uncomfortable property it is acquainted with. To say it pointedly: Remove this property and you remove the phenomenal quality. And remove the phenomenal quality and you remove the whole pain-state. And since, by definition, the uncomfortable property a subject is acquainted with in being in a pain-state is just a pain, we have to conclude that it cannot be for a subject as if it has a pain in the absence of a pain it is acquainted with. And this is exactly what the PMDP in the literal reading says. Well, this reasoning will not remain undisputed. Let us take a brief look at the most prominent objections. Something like the standard objection is familiar from the debate about visual experiences. Conveyed to the context at hand, it can be stated as follows: There may be a sense in which it is appropriate to talk of pains even in cases like $\mathrm{SP}_{1}$, but we are not entitled to infer from the appropriateness of this talk to the existence of real pains in such cases. The pains one is talking about in such cases are 'merely apparent' or 'merely intentional' pains and as such they have no existence whatsoever. In other words: Talking about them means nothing 
but talking about what the relevant pain-states are about. And the absence of pains does not prevent these pain-states from being about pains.

This objection is misplaced. While it makes sense - even if this sense may differ from the one proponents of the objection have in mind - to say that, for example, Macbeth was aware of a merely apparent dagger, it makes absolutely no sense to say that James in case $\mathrm{SP}_{1}$ feels a merely apparent pain. For, according to our concept of a pain, James' pain in case $\mathrm{SP}_{1}$ is as real as a pain can be. It exists no more and no less than the pains of any other person ever having a pain in her knee. So we have to conclude that, when it comes to pain, the standard objection has no force.

Further objections concern the fact that my argument contains an inference from the conceptual truth that, according to our concept of pain, there is a pain if and only if it is a certain way for a subject to the ontological conclusion that there is a pain if and only if it is this way for a subject. There are two serious objections one might raise against this inference. $^{13}$

The first rests on the following two assumptions. The relevant conceptual truth is an a priori truth and the concept of pain is such that the ontological questions of what pains are and when there are pains can be answered only a posteriori. I concede the first assumption, but deny the second. The idea behind the second assumption is, of course, that the concept of pain is a natural kind concept like water or light. These concepts are such that how something has to be in order to fall under them depends on contingent features of the actual world. If the concept

\footnotetext{
${ }^{13}$ Among the objections I do not take seriously are those that rest upon assumptions along the following lines: Conceptual truths are in principle irrelevant for answering metaphysical questions since in metaphysics one is concerned with the things themselves and not with the concepts of these things. The mistake in this assumption should be obvious: Even metaphysical questions need to be asked before they can be answered. But in asking a question one cannot but make use of certain concepts. And it is these concepts which determine, together with the state of the world, what counts as a true answer to the question.
} 
of pain were a concept of this kind, the second assumption would indeed be true. I take it, however, to be shown by others that the concept of pain is not a concept of this kind (see e.g. Chalmers 2003 or Kripke 1980).

While the first objection is much-discussed, the second is frequently overlooked by proponents of the view defended here. It runs as follows: It is a well-known truth that concepts, especially concepts that are not natural kind concepts, can be empty. For this reason the question of whether there are pains (in the sense of the concept analyzed) cannot be answered through a conceptual analysis alone. So, given that the ontological conclusion of the argument under consideration is meant to be about pains in the actual world, an affirmative answer to this question is essential for this argument. But, on the face of it, there are strong reasons to doubt the truth of an affirmative answer. There is an initial suspicion that if pains were as our concept of pain says they are, they would be rather strange entities. And, given the reasoning in the reminder of this paper will be correct, this suspicion will be confirmed. For, according to the pain-thesis, which I defend, pains are properties of sense-data. Is this not reason enough to assume that the corresponding concept of pain is empty?

This is, no doubt, a serious objection. I accept the commitment to the non-emptiness of the relevant concept of pain and I concede that the above argument against this assumption has some weight. But I also insist that it is outweighed by an even stronger argument to the contrary. To put it straightforwardly: Our relation to the entities we take to be our own pains is not of a kind that allows for systematic error in applying an everyday concept to them. Just think about any situation you would describe as a situation of having a pain. Could it really be that you systematically err regarding the question of whether there is an uncomfortable quality of the right kind present to you? I guess it is not. ${ }^{14}$ Hence, we should better not assume that

\footnotetext{
${ }^{14}$ To be sure, stating this fact is one thing, explaining it is a completely different matter. But addressing this latter task goes beyond the scope of this paper.
} 
our application of our everyday concept of pain is systematically fallacious - not even in face of cases like $\mathrm{SP}_{1}{ }^{15}$ So, we can conclude that none of the objections gives us sufficient reason to doubt the truth of PMDP.

Having settled this, we can take PMDP as a condition of adequacy for the accounts of painstates outlined above. Let us consider how well these accounts perform with respect to this condition. To begin with, the truth of PMDP poses a prima facie problem for naïve painrealism as well as for pain-intentionalism since both accounts contain the assumption that a pain is the kind of physical property (damage to a body-part) which is absent in situations like $\mathrm{SP}_{1}$ while the $\mathrm{PMDP}$ explicitly denies that pains are absent in situations like $\mathrm{SP}_{1}$. And in the case of naive pain-realism, this prima facie problem quickly turns into a decisive objection. Since naive pain-realism takes the relevant physical properties to be real constituents of painstates it lacks any resources to deal with the problem. ${ }^{16}$ So, let us immediately come to painintentionalism. Recall:

According to pain-intentionalism, pains are

(i) physical, public properties (presumably damage to body-parts) that are

(ii) represented by pain-states.

\footnotetext{
${ }^{15}$ It is important to note that nothing hinges on the assumption that it is our everyday concept which meets the conditions established in the discussion of both objections (being not a natural kind concept and being not empty). All that matters is that there is $a$ concept of pain which meets both conditions.

${ }^{16}$ Note in this connection that, given the truth of PMDP, the popular disjunctivist move is no option. While a disjunctivist about pain-states would maintain that ordinary pain-states and phantom-limb pain-states are states of different fundamental kinds insofar as only the former goes along with a pain, PMDP explicitly says that there is a pain in cases of phantom-limb pain-states.
} 
Does pain-intentionalism have the resources to do justice to PMDP? Even though it also contains the assumption that a pain is damage to a body-part, its prospects are indeed significantly better.

In the relevant literature we find two proposals that, at first appearance, seem to have the potential to meet this requirement. I shall call them, for want of any better expressions, relationalism and platonism. Both are usefully spelled out by Michael Tye:

„The term 'pain' [...] applies to the quality represented insofar as (and only insofar as) it is within the content of a pain experience.“ (Tye 2006a, p. 101)

„Suppose I feel a pain in a leg that no longer exists. It seems to me that I have a pain, and I am conscious of a quality it is correct to call 'pain' [...]. But the quality has no bearer. My experience represents that there is tissue damage in my leg, or so I say. But there is no tissue damage and no leg. My pain experience is a hallucination even though I am certainly conscious of pain. [my italics]“ (Tye 2006b, p. 169)

The proposal put forward in the first passage is relationalism. Tye holds to the intentionalist assumption that a pain is damage to a body-part, but adds a qualification: Put in our terminology, a pain is damage to a body-part insofar as and only insofar as it is represented through a pain-state. The idea spelled out in the second passage is platonism. According to platonism, the subject of a phantom-limb pain is acquainted with the same property, a pain, that it would be acquainted with if it were in a corresponding pain-state under normal conditions. But, in contrast to the latter kind of case, the property is not instantiated in any object - be it the relevant body-part or a representation thereof. Rather it is a uninstantiated property. ${ }^{17}$

\footnotetext{
${ }^{17}$ Note that the idea of platonism is significantly different from the assumption, discussed some paragraphs earlier, that the relevant pains are merely apparent and have no ontological status whatsoever. That is not what
} 
When we consider these proposals with regard to situations like $\mathrm{SP}_{1}$ and $\mathrm{SP}_{2}$, we find that, taken in isolation, both can do justice to one kind of situation, at least to some extent, but fail to do so to the other. Relationalism does justice to situations like $\mathrm{SP}_{2}$, situations in which there is no pain even though there is damage to a body-part that would have brought about a pain-state if the conditions were normal. Its Proponents can offer the following explanation: Damage to a body-part as such is not necessarily a pain. In order to be a pain it has to be represented by a pain-state. And since this latter condition is not met in situation $\mathrm{SP}_{2}$, there is no pain in this situation. ${ }^{18}$ When it comes to situations like $\mathrm{SP}_{1}$, however, proponents of relationalism have no explanation to offer. In situations like $\mathrm{SP}_{1}$ there are pains even though corresponding damage to body-parts are missing. But, even according to relationalism, every pain is necessarily damage to a body-part. And as long as one understands this claim in the usual way, ${ }^{19}$ relationalism is incompatible with situations like $\mathrm{SP}_{1}$. Proponents of platonism, on the other hand, can offer an explanation of the possibility of situations like $\mathrm{SP}_{1}$. According to them, it is, in a highly specific sense, not correct to say that there is no damage to a body-part in this situation. There is one, but it is not, as it would be the case under normal conditions, instantiated in the body of the subject. Instead it is a

platonism says. According to platonism, the relevant pains are perfectly real pains with a well-defined ontological status. They are uninstantiated properties. It is just that their ontological status is somewhat special.

${ }^{18}$ It should not remain unnoticed, however, that this explanation is somewhat counterintuitive. According to it, a pain is a pain in virtue of a contingent, relational property - the property of being represented in a pain-state. This implies that the very same instance of a property which was a pain up to a certain point of time can, after this point of time, continue to exist without being a pain any more. It just has to cease to be represented by a pain-state. This seems to square badly with the intuitions lying behind PMDP. If we think about a pain we are strongly inclined to assume that the very same property-instance which is this pain cannot continue to exist without being a pain any more. It seems to be a pain essentially.

${ }^{19}$ Understanding the claim in the usual way means here, amongst other things, not to understand it in the way suggested by platonism (see below). 
uninstantiated property. And this uninstantiated property is the pain felt by the subject in the situation. In the end, however, platonism is no better off than relationalism. For, in contrast to the latter, platonism cannot account for situations like $\mathrm{SP}_{2}$. Nothing in this view explains why damage to a body-part that is not represented by a pain-state is not a pain.

There is, however, an obvious way to compensate for the weaknesses of both proposals: One just has to combine platonism with relationalism. Roughly put, on the resulting account, one would have no pain-states without pains in virtue of platonism and no pains without painstates in virtue of relationalism. This idea appears promising at first glance. But, unfortunately, it cannot be made to work since platonism is untenable for independent reasons. Note first that uninstantiated properties are abstract objects. Then consider the attributes commonly used to characterize abstract objects. Künne, for example, mentions, amongst others, non-observability $<$ Nicht-Wahrnehmbarkeit>, uniqueness <Einzigkeit>, nonspatiality <Nicht-Räumlichkeit> and non-temporality <Nicht-Zeitlichkeit> (Künne 1983, p. 63). Is it really believable that entities that have these properties could be present to us in roughly the same way pains are present to us in ordinary pain-states? I think it is not. Whatever it is that is present to us in such states, it exists presumably in space and certainly in time. And, as a consequence, at least the objects of some phantom-limb pain-states must be numerically different from each other. So, it seems as if they are neither non-spatial nor nontemporal nor unique. And, as far as non-observability is concerned, it would be surprising, to say the least, if whatever makes universals non-observable were not also preventing them from being objects of our acquaintance. Hence, platonism as well as any view comprising platonism is to be rejected. $^{20}$

\footnotetext{
${ }^{20}$ To be honest, to me the failure of platonism seems to be quite evident. However, the relative popularity of the view (see e.g. Dretske 1999 or Johnston 2004) as well as the significant shortage of serious critique of it (but see Kriegel 2011 and Pautz 2007 for exceptions) indicates otherwise. So maybe my judgment is too rash.
} 
The conclusion to be drawn is that pain-intentionalism can do justice to PMDP only at the price of ending up in a position that is inacceptable for independent reasons. As a consequence, we are left with the last alternative available: indirect pain-realism. Recall:

According to indirect pain-realism, pains are

(i) properties of sense-data and, in addition to that,

(ii) representations of physical, public properties (presumably damage to body-parts).

Since sense-data are, so to speak, mind-dependent by nature, there is no doubt that indirect pain-realism meets PMDP perfectly well. ${ }^{21}$ Given the problems that naïve pain-realism and pain-intentionalism have in meeting the same requirement, this is a very strong argument in favour of indirect pain-realism and, thus, for the pain-thesis. And, in the absence of a comparably strong argument against the latter thesis, we can take it to be confirmed - at least until further consideration. ${ }^{22}$

With this the main work of this paper is done. Both premises of the argument from pain are confirmed and, thanks to the validity of the argument, the same is true of its conclusion. Recall:

Being in a visual experience is to be acquainted with a sense-datum too.

Nonetheless, the force of the argument may still not be fully apparent. This is due to the fact that the significance of the reasoning for the analogy-thesis is clearly visible only before the

\footnotetext{
${ }^{21}$ In this sense Murat Aydede is perfectly right when he states: „Sense-datum theories seem to be tailormade for the peculiarities of pain experiences [...].” (Aydede 2006, p. 8)

${ }^{22}$ To be sure, I have done nothing to show that there is no such argument. And, indeed, I am not sure about this. But I leave it to others to look for such an argument.
} 
background of the reasoning for the pain-thesis. Hence, it is only now that we are in the position to work out this significance in full clarity.

Throughout my reasoning for the pain-thesis I have addressed the question of what it means to say that it is for a subject as if it has a pain. The answer was based on an assumption defended in section 2, the assumption that pain-states are transparent, that is, that the way it is like for a subject to be in a pain-state is fully exhausted by the uncomfortable property present to that subject. This transparency of pain-states makes it impossible that it is somehow for a subject to be in a pain-state even though there is (in a sense yet to be determined) no uncomfortable property present to it. For without this property there is no phenomenal quality and, thus, no pain-state at all. Furthermore, I emphasized that the following holds for conceptual reasons: Every uncomfortable property present to a subject which brings about the phenomenal quality specific for pain-states is a pain - not an apparent or an intentional pain or something like that, but a perfectly real pain. That is, for an uncomfortable quality to be a real pain no more and no less is needed than what is needed to bring about the phenomenal quality specific for pain-states. Finally, I argued that there is only one way to account for occurrences of real pains in the absence of damage to body-parts: One must ascribe to them, or at least some of them, the ontological status of sense-data. The outcome of this reasoning is highly remarkable: Given (i) the transparency of pain-states and (ii) the possibility of pains in the absence of relevant physical properties, the fact that pain-states have the phenomenal quality they have is a sufficient condition for the existence of a certain kind of sense-data, namely pains.

Now consider, in the light of this outcome, the analogy-thesis once again. The analogy-thesis states that all the conditions, which are in the case of pains together sufficient for the existence of sense-data, have an analog in the case of visual experiences. Visual experiences are (i) transparent and (ii) can have their phenomenal qualities in the absence of relevant physical properties. Furthermore, there is no reason to assume that this is true of visual 
experiences in any other sense than it is true of pain-states. Take, say, a hallucination of something red: Just as being in a pain-state means nothing but being acquainted with a certain kind of uncomfortable property, having such a hallucination means nothing but being acquainted with a certain kind of sensible redness. And, just as the way it is like for a subject to be in a pain-state is fully exhausted by the uncomfortable property present to the subject of the state, the way it is like for a subject to have a hallucination of something red is fully exhausted by the sensible redness the subject is acquainted with. There is nothing about the pain in a phantom-limb case that makes it more real than the sensible redness present to the subject of a hallucination of something red. But, and this is the decisive point, if in cases of pains these conditions are sufficient to establish the existence of a certain kind of sense-data, we cannot but conclude the same for cases of visual experiences. We cannot but conclude that, for example, in our case of a hallucination as of something red these conditions are sufficient to establish the existence of a certain kind of sense-datum - a sense-datum which brings about the phenomenal quality specific for visual experiences as of something red. And this is precisely what the conclusion of the argument from pain says.

With this reasoning clearly in view, we are in the position to face an objection which, presumably, is already preying upon the reader's mind: The analogy-thesis can be true only if there are principles analogous to PMDP holding for cases of visual experiences. Apparently, such a principle would have to be something along the following lines:

For any subject it is impossible that (i) it is for them as if there is an instance of redness before them while, in fact, there is none and that (ii) there is an instance of redness before them while it is not for them as if there is one.

But is this not plainly wrong? 
This looks like a strong objection. But it isn't. Let us grant that (i) it is a condition of the truth of the analogy-thesis that there are principles analogous to the PMDP holding for visual experiences and their objects and that (ii) the above principle is false if it is understood according to our everyday language. But, even put together, both assumptions fail to provide an effective argument against the analogy-thesis.

Note first that both assumptions are fully compatible with indirect realism. If principle is false if it is understood according to our everyday language "redness” denotes physical redness, the redness of ordinary, physical objects. Indirect realists concede the falsehood of the principle in this reading (assumption (ii)), but insist that it has a second reading in which it is true. In this reading "redness" denotes not ordinary physical redness, but what we may call sensible redness, the kind of redness only sense-data have. And it is the principle in this latter reading, which is, according to indirect realism, the analog to PMDP for cases of visual experiences (assumption (ii)).

To be sure, when it comes to the question of whether both assumptions provide an effective argument against the analogy-thesis, we should better not rest our case on assumptions made on the basis of indirect realism. But we don’t need to do so in order to reject the objection. Note that the conjunction of both assumptions speaks against the analogy-thesis only under the presupposition that physical properties, like physical redness, are the best candidates available to be the elements in cases of visual experiences which are analogous to pains in cases of pain-states. But just this presupposition is unjustified - at least in the context of the current discussion. For whether or not physical properties, like physical redness, are the best candidates available to be the elements in cases of visual experiences analogous to pains, depends on whether or not cases of visual experiences are analogous in the relevant respects to cases of pain-states (i.e. on the truth value of the analogy-thesis) - not the other way around. Hence, in order to respect the order of logical priority one first has to consider the analogy-thesis through a careful investigation of cases of pain-states and cases of visual 
experiences and then decide whether there are better candidates for the role of elements analogous to pains than physical properties, like physical redness. This is exactly what we did. And since we found that the analogy-thesis is true and that, hence, there are better candidates, we can reject the relevant presupposition - and with it the objection resting upon it.

Finally, let us reconsider the claim made at the beginning of the paper, that the argument from pain is stronger than the good old argument from hallucination. The reason is now apparent. It has to do with the different resources provided by our everyday language about cases of visual experiences and cases of pain-states. In our everyday language there is no established talk about objects of visual experiences which are ontologically dependent on these experiences. Our everyday talk about objects of visual experiences is exclusively about physical objects. When it comes to pain, however, the situation is completely different. Our everyday pain-talk is guided by PMDP. That is, there is not only well-established talk about objects of pain-states which are ontologically dependent on these states; this is also the only well-established talk about objects of pain-states. This is a very strong reason to believe in such objects and it finally persuaded us to accept the pain-thesis. But it is precisely this reason that is absent when it comes to cases of visual experiences. If one denies the existence of sense-data of visual experiences, no conflict with our everyday talk arises. This is why, as I put it above, the pain-thesis is considerably easier to defend than the corresponding premise of the argument from hallucination. And this, in turn, is why the argument from pain is stronger than the argument from hallucination.

Admittedly, the above line of reasoning commits us to the assumption that it is possible in principle to introduce terms for sensible properties of visual experiences which are guided by a principle analogous to PMDP. But we can happily accept this commitment. Take e.g. our everyday term for physical redness. We could quite easily introduce a term which is related to this term roughly in the way our term "pain” is related to terms like "wound”, "injury” and so on. Put simply, we just have to suspend the appearance/reality-distinction incorporated in the 
rules that guide our use of this term. The emerging term would be correctly applied if and only if one has a red-experience just as "pain" is correctly applied if and only if one is in a pain-state.

\section{Conclusion}

I've presented a valid argument for indirect realism and I've defended its premises with some effort. Nonetheless there are surely ways to counter my argument. And I do not hope to have converted even one opponent of indirect realism. What I hope to have shown however, is that the argument from pain is an improvement of the argument from hallucination and that it is a serious argument in its own right. Given I were successful in that, my paper may contribute to re-establishing indirect realism as a serious alternative in the philosophy of perception.

\section{References}

Aydede, Murat (2006). Introduction: A Critical and Quasi-Historical Essay on Theories of Pain. In Aydede (Ed), Pain: New Essays on Its Nature and the Methodology of Its Study (pp. 1-58). Cambridge, Massachusetts, London: MIT Press.

Brewer, Bill (1995). Bodily Awareness and the Self. In Bermudez, Jose Luis; Eilan, Naomi and Marcel, Anthony (Ed), The Body and the Self (pp. 291-309). Cambridge, Massachusetts, London: MIT Press.

Castañeda, Hector-Neri (1977). Perception, Belief, and the Structure of Physical Objects and Consciousness. Synthese, 35 (3), 285 - 351.

Casullo, Albert (1986). The Spatial Structure of Perceptual Space. Philosophy and Phenomenological Research 46 (June), 665-671.

Chalmers, David (2003). The Content and Epistemology of Phenomenal Belief, In Aleksandar Jokic, Quentin Smith (Ed), Consciousness: New Philosophical Perspectives (pp. 220-272), Oxford, New York: Oxford University Press. 
Dretske, Fred (1999). The Mind's Awareness of Itself. Philosophical Studies, 95 (1-2), 103-24.

[Author's name] (forthcoming).

Harman, Gilbert (1990). The Intrinsic Quality of Experience. Philosophical Perspectives, 4, 31-52.

Hill, Christopher (2006), Ow! The Paradox of Pain. In: Aydede (Ed), Pain: New Essays on Its Nature and the Methodology of Its Study (pp. 75-98). Cambridge, Massachusetts, London: MIT Press.

Johnston, Mark (2004). The Obscure Object of Hallucination. Philosophical Studies, 120 (1-3), 83-113.

Kriegel, Uriah (2011). The Veil of Abstracta. Philosophical Issues. 21 (1), 245-267.

Kripke, Saul (1980). Naming and Necessity. Cambridge Massachusetts: Harvard University Press.

Künne, Wolfgang (1983). Abstrakte Gegenstände. Frankfurt a.M.: Vittorio Klostermann.

Martin, Michael G.F. (2002). The Transparency of Experience. Mind and Language, 4 (4), 376-425.

Pautz, Adam (2007). Intentionalism and Perceptual Presence. Philosophical Perspectives 21 (1), 495-541.

Rowlands, Mark (2001). The Nature of Consciousness. Cambridge: Cambridge University Press.

Thompson, Brad (2008). Representationalism and the Argument From Hallucination. Pacific Philosophical Quarterly, 89 (3), 384-412.

Tye, Michael (2006a). Another Look at Representationalism about Pain. In: Aydede (Ed), Pain: New Essays on Its Nature and the Methodology of Its Study (pp. 99-120). Cambridge, Massachusetts, London: MIT Press.

Tye, Michael (2006b). In Defense of Representationalism: Reply to Commentaries. In: Aydede (Ed), Pain: New Essays on Its Nature and the Methodology of Its Study (pp. 163-175). Cambridge, Massachusetts, London: MIT Press.

Dirk Franken

University of Marburg

Wilhelm-Röpke-Straße $6 b$

35032 Marburg (Germany) 